\title{
An elementary construction of the geometric algebra
}

\author{
Alan Macdonald \\ Department of Mathematics, Luther College \\ Decorah IA, 52101 U.S.A. \\ macdonal@luther.edu
}

March 30, 2016

\begin{abstract}
We give a simple, elementary, direct, and motivated construction of the geometric algebra over $\mathbf{R}^{n}$.
\end{abstract}

Mathematics Subject Classification 2000. Primary 15A66.

Keywords: Geometric Algebra, Clifford Algebra.

1. Introduction. We give here a new construction of the geometric algebra $\mathrm{GA}(n)$ over $\mathbf{R}^{n}$ with the standard inner product. (We then extend to an inner product of arbitrary signature.) A construction of $\mathrm{GA}(n)$ proves that a structure satisfying the axioms of $\operatorname{GA}(n)$ exists. Simply stating the axioms and proceeding, as is commonly done, is a practical approach. But then there is no guarantee that $\mathrm{GA}(n)$ exists, as the axioms might be inconsistent. A mathematically complete presentation must show that $\mathrm{GA}(n)$ exists.

One can consider the construction given here as the latest step in the well known sequence of constructions of number systems based on the natural numbers: the integers are equivalence classes of ordered pairs of natural numbers, the rational numbers are equivalence classes of ordered pairs of integers, the real numbers are Dedekind cuts or equivalence classes of Cauchy sequences of rational numbers, and the inner product space $\mathbf{R}^{n}$ consists of $n$-tuples of real numbers [3]. Each step in the sequence uses only the previously constructed system. (It is a stretch to call $\mathbf{R}^{n}$ a "number system", but it becomes one when the geometric algebra structure is imposed on it. Then we do not need to construct the standard complex numbers or quaternions, as they are embedded in $\mathrm{GA}(2)$ and $\mathrm{GA}(3)$, respectively.)

My goal is to provide a construction of $\mathrm{GA}(n)$ suitable for someone with only an elementary knowledge of $\mathbf{R}^{n}$. The construction is simple, elementary, direct, and motivated. Two features of the construction help make this so.

First, the construction uses only elementary properties of $\mathbf{R}^{n}$. Some constructions use more advanced mathematical structures, such as tensor products. 
(Our construction is compared to others in Section 7.) The geometric algebra $\mathrm{GA}(n)$ is a fundamental mathematical structure which is part of the essence of Euclidean space. It therefore deserves a construction not using less fundamental structures than $\mathbf{R}^{n}$.

Second, the construction is based directly upon the two fundamental identities which distinguish $\mathrm{GA}(n)$ : If $e$ and $f$ are orthonormal vectors in $\mathbf{R}^{n}$, then

$$
\begin{aligned}
e e & =1, \\
e f & =-f e .
\end{aligned}
$$

Eqs. (1) and (2) motivate our construction.

$\mathrm{GA}(n)$ is a $2^{n}$ dimensional vector space containing $\mathbf{R}^{n}$ which is also an associative algebra with identity. Its vectors are called multivectors. In addition, Eqs. (1) and (2) are satisfied. We list the vector space and algebra axioms for reference. Let $a$ and $b$ be scalars and $u, v$, and $w$ be multivectors. Then

$$
\begin{array}{lrl}
\text { V1. } u+v=v+u . & \text { A1. } u(v w)=(u v) w . \\
\text { V2. }(u+v)+w=u+(v+w) . & \text { A2. } u(v+w)=u v+u w, \\
\text { V3. } u+0=u . & & (v+w) u=v u+w u . \\
\text { V4. } u+(-u)=0 . & \text { A3. }(a u) v=u(a v)=a(u v) . \\
\text { V5. } 1 u=u . & \text { A4. } 1 u=u 1=u . \\
\text { V6. } a(b u)=(a b) u . & \\
\text { V7. } a(u+v)=a u+a v . & \\
\text { V8. }(a+b) u=a u+b u . &
\end{array}
$$

2. The lemma. Let $\left\{e_{1}, e_{2}, \ldots, e_{n}\right\}$ be an orthonormal basis for $\mathbf{R}^{n}$. If we are to be able to multiply an arbitrary number of vectors, then we must be able to form sequences of $e$ 's:

$$
E=e_{i_{1}} e_{i_{2}} \cdots e_{i_{r}}
$$

A given $e$ may occur more than once, and there is no specified order for the $e$ 's. Denote the empty sequence by 1 . For now, the sequences are uninterpreted symbols, but they will become products.

Let $E^{\prime}$ be another sequence of the form Eq. (3), which is obtained from $E$ by (i) exchanging pairs of adjacent and unequal $e$ 's in $E$, and (ii) inserting and deleting pairs of adjacent and equal e's. Write $T(E)=E^{\prime}$. Call $T$ even or odd according as $N_{e}$, the number of exchanges in $T$, is even or odd.

If Eqs. (1) and (2) are to be true, then we need to set $E^{\prime}= \pm E$, according as $T$ is even or odd. There is however a question of consistency, as many $T$ s transform $E$ to $E^{\prime}$. Will $T$ be always even or always odd? The answer is yes:

LEMma. For given $E$ and $E^{\prime}$, all $T$ with $T(E)=E^{\prime}$ are even or all are odd.

Proof. Let $G(E)$ be the number of times a subscript in $E$ is greater than a subscript to its right. For example, $G\left(e_{2} e_{1} e_{5} e_{5} e_{4}\right)=3$. Exchanging a pair of adjacent and unequal $e$ 's in $E$ changes $G$ by \pm 1 , and so changes the parity of $G$, i.e., changes $G$ from even to odd or odd to even. Inserting or deleting a pair of adjacent and equal $e$ 's changes $G$ by an even number, and so does not change its parity. Thus $T$ is even or odd according as $G(E)$ and $G\left(E^{\prime}\right)$ have the same or different parity. 
3. Construction of the vector space $\mathbf{G A}(n)$. If there is an even $T$ with $T(E)=E^{\prime}$, set $E^{\prime}=E$ and $-E^{\prime}=-E$. For example, $e_{2} e_{3} e_{1} e_{3}=$ $e_{1} e_{2}$. Eq. (1) is a special case: $e_{i} e_{i}=1$. The relation " $=$ " is an equivalence relation. For example, it is symmetric. For if $E^{\prime}=E$, then there is an even $T$ with $T(E)=E^{\prime}$. Then $T^{-1}\left(E^{\prime}\right)=E$. (For $T^{-1}$ undo, in reverse order, the exchanges, insertions, and deletions of $T$.) Since $T^{-1}$ is even, $E=E^{\prime}$. Similarly, if $-E^{\prime}=-E$, then $-E=-E^{\prime}$.

If there is an odd $T$ with $T(E)=E^{\prime}$, set $E^{\prime}=-E$ and $-E=E^{\prime}$. For example, $e_{3} e_{2} e_{1} e_{3}=-e_{1} e_{2}$. Eq. (2) is a special case: $e_{i} e_{j}=-e_{j} e_{i}$ for $i \neq j$. The relation "=" remains an equivalence relation. For example, it remains symmetric by definition.

The negatives of the members of an equivalence class form another class: $\left(E^{\prime}=E\right) \Leftrightarrow\left(-E^{\prime}=-E\right)$ and $\left(E^{\prime}=-E\right) \Leftrightarrow\left(-E^{\prime}=E\right)$.

The vector space $\operatorname{GA}(n)$ is the set of linear combinations of the equivalence classes. Take the classes to be linearly independent, except for a class and its negative. The vector space axioms V1-V8 are automatically satisfied. ${ }^{1}$

Each equivalence class has a unique member $\pm B$, where $B$ is of the form

$$
B=e_{i_{1}} e_{i_{2}} \cdots e_{i_{r}}, i_{1}<i_{2}<\cdots<i_{r} .
$$

Those equivalence classes containing a $B$ form a basis for the vector space $\mathrm{GA}(n)$. There are $2^{n}$ such sequences (each of the $n$ e's can appear or not). Thus the dimension of $\mathrm{GA}(n)$ is $2^{n}$.

4. Construction of the algebra $\mathbf{G A}(\mathbf{n})$. Let $E=e_{i_{1}} e_{i_{2}} \cdots e_{i_{r}}$ and $F=e_{j_{1}} e_{j_{2}} \cdots e_{j_{s}}$. Define the geometric product of $E$ and $F$ :

$$
E F=\left(e_{i_{1}} e_{i_{2}} \cdots e_{i_{r}}\right)\left(e_{j_{1}} e_{j_{2}} \cdots e_{j_{s}}\right)=e_{i_{1}} e_{i_{2}} \cdots e_{i_{r}} e_{j_{1}} e_{j_{2}} \cdots e_{j_{s}} .
$$

The product is well defined on equivalence classes: If $E=E^{\prime}$ and $F=F^{\prime}$, then $E F=E^{\prime} F^{\prime}$.

The product is obviously associative. Thus we can now consider the sequences in Eq. (3) to be products of $e$ 's with parentheses removed.

Extend the geometric product by linearity to all of $\mathrm{GA}(n)$ :

$$
\left(\sum_{i} a_{i} E_{i}\right)\left(\sum_{j} b_{j} F_{j}\right)=\sum_{i, j} a_{i} b_{j} E_{i} F_{j} .
$$

The product remains well defined.

\footnotetext{
${ }^{1}$ Note added 13 May, 2007.

The vector space $\mathrm{GA}(n)$ consists of formal linear combinations of the equivalence classes, making identifications demanded by vector space operations (e.g., $2 e_{1}+4 e_{1}=6 e_{1}$ ) and by the requirement that $E+(-E)=0$ (e.g., $e_{1} e_{2}+e_{2} e_{1}=0$ ).

More technically, let $F$ be the free vector space over the equivalence classes. (See, for example, http://planetmath.org/?op=getobj\&from=objects\&id=4196.) And let $Z$ be the subspace of $F$ spanned by elements of the form $E+(-E)$. Then $G A(n)=F / Z$.

Our modding out by $Z$ is similar to some constructions of the tensor product of vector spaces. For example, see http://www.math.harvard.edu/ tomc/math25/tensor.pdf.
} 
The geometric product defined by Eq. (5) satisfies axioms A1-A4.

5. Other orthonormal bases. Even though we constructed GA $(n)$ using $\left\{e_{i}\right\}$, this orthonormal basis is not distinguished in $\operatorname{GA}(n)$ : If $\left\{f_{i}\right\}$ is another orthonormal basis, then the $f$ 's have the same properties as the $e$ 's. To see this, let $f_{r}=\sum_{j} a_{r j} e_{j}, r=1,2, \ldots, n$. Then

$$
\begin{aligned}
f_{r} f_{s} & =\sum_{j, k} a_{r j} a_{s k} e_{j} e_{k} \\
& =\sum_{j=k} a_{r j} a_{s k} e_{j} e_{k}+\sum_{j \neq k} a_{r j} a_{s k} e_{j} e_{k} \\
& =f_{r} \cdot f_{s}+\sum_{j<k}\left(a_{r j} a_{s k}-a_{s j} a_{r k}\right) e_{j} e_{k} .
\end{aligned}
$$

Since the $f_{i}$ are orthonormal, $f_{r} f_{r}=1$ and $f_{r} f_{s}=-f_{s} f_{r}$ for $r \neq s$. This establishes Eqs. (1) and (2) for the $f$ 's.

Products of the $f$ 's span $\mathrm{GA}(n)$, since the $e$ 's in the basis elements Eq. (4) can be expressed in terms of the $f$ 's. It follows that the $2^{n}$ products

$$
f_{i_{1}} f_{i_{2}} \cdots f_{i_{r}}, i_{1}<i_{2}<\cdots<i_{r}
$$

are linearly independent.

We prove that $f_{i_{1}} f_{i_{2}} \cdots f_{i_{r}}$ above is an $r$-vector with respect to the $e^{\text {'s. }}{ }^{2}$ First consider the reflection $e_{i} \rightarrow g_{i}=-v e_{i} v$ for some unit vector $v$. Then:

$$
g_{i_{1}} g_{i_{2}} \cdots g_{i_{r}}=\left(-v e_{i_{1}} v\right)\left(-v e_{i_{2}} v\right) \cdots\left(-v e_{i_{r}} v\right)= \pm v e_{i_{1}} e_{i_{2}} \cdots e_{i_{r}} v .
$$

I claim that $g_{i_{1}} g_{i_{2}} \cdots g_{i_{r}}$ is an $r$ vector. For ease of notation, we take a specific case which shows all of the possibilities:

$$
\begin{aligned}
g_{1} g_{2} & =\left(a_{1} e_{1}+a_{2} e_{2}+a_{2} e_{3}+a_{4} e_{4}\right)\left(e_{1} e_{2}\right)\left(a_{1} e_{1}+a_{2} e_{2}+a_{2} e_{3}+a_{4} e_{4}\right) \\
& =\left(e_{1} e_{2}\right)\left(-a_{1} e_{1}-a_{2} e_{2}+a_{3} e_{3}+a_{4} e_{4}\right)\left(a_{1} e_{1}+a_{2} e_{2}+a_{3} e_{3}+a_{4} e_{4}\right) .
\end{aligned}
$$

The terms in $-a_{1} e_{1}-a_{2} e_{2}+a_{3} e_{3}+a_{4} e_{4}$ whose index is in the set $\{1,2\}$ of indices in $e_{1} e_{2}$ appear with the opposite sign from those whose index is in $\{3,4\}$. Multiply the two vectors on the right side of Eq. (7). Then multiply the terms of the product by the bivector $e_{1} e_{2}$. We show that the result is a bivector.

The "diagonal" terms of the product, e.g., $-\left(a_{1} e_{1}\right)\left(a_{1} e_{1}\right)=-a_{1}^{2}$, are scalars. Multiply them by $e_{1} e_{2}$. The result is a bivector. Products with indices both from $\{1,2\}$ or both from $\{3,4\}$, like $-\left(a_{1} e_{1}\right)\left(a_{2} e_{2}\right)$ and $\left(a_{3} e_{3}\right)\left(a_{4} e_{4}\right)$, are paired with their negatives $-\left(a_{2} e_{2}\right)\left(a_{1} e_{1}\right)$ and $\left(a_{4} e_{4}\right)\left(a_{3} e_{3}\right)$. When added, they produce 0 . The remaining terms have one index from $\{1,2\}$ and one from $\{3,4\}$, e.g., $-\left(a_{1} e_{1}\right)\left(a_{3} e_{3}\right)$ and $\left(a_{3} e_{3}\right)\left(a_{2} e_{2}\right)$. Multiply them by $e_{1} e_{2}$. The result is a bivector.

The orthogonal transformation $e_{i} \rightarrow f_{i}$ is a composition of such reflections. Applying the above argument successively to each reflection shows that $f_{i_{1}} f_{i_{2}} \cdots f_{i_{r}}$ is an $r$-vector.

\footnotetext{
2 This material added 10/22/07, modified $11 / 22 / 07$ and 5/27/08.
} 
6. Other signatures. Let $\mathbf{R}^{p, q}$ be an inner product space with orthonormal basis $\left\{e_{i}\right\}$, where $e_{i} \cdot e_{i}=1$ for $1 \leq i \leq p$ and $e_{i} \cdot e_{i}=-1$ for $p<i \leq p+q=$ $n$. We extend our construction of $\mathrm{GA}(n)$ over $\mathbf{R}^{n}$ to the geometric algebra $\mathrm{GA}(p, q)$ over $\mathbf{R}^{p, q}$. In $\mathrm{GA}(p, q)$ the vector space axioms $\mathrm{V} 1-\mathrm{V} 8$, the algebra axioms A1-A4, and Eq. (2) are unchanged. Only Eq. (1) changes, becoming $e e=e \cdot e(= \pm 1)$.

To accommodate this change, we need only extend the definition of even and odd $T$. Let $T(E)=E^{\prime}$. Recall that $N_{e}$ is the number of exchanges in $T$. The lemma states that the parity of $N_{e}$ depends only on $E$ and $E^{\prime}$. Let $N_{i}$ be the net gain from $T$ of the number of pairs of equal $e$ 's with $e \cdot e=-1$, due to insertions and deletions of adjacent such pairs. Then $N_{i}$ depends only on $E$ and $E^{\prime}$. Thus the parity of $N_{e}+N_{i}$ depends only on $E$ and $E^{\prime}$. Call $T$ even or odd according as $N_{e}+N_{i}$ is even or odd. We have just proved that all $T$ with $T(E)=E^{\prime}$ are even or all are odd. This extends the lemma to inner product spaces of arbitrary signature.

With the lemma extended, the construction of $\mathrm{GA}(p, q)$ proceeds verbatim as for $\operatorname{GA}(n)$. However, some of the examples change. In particular, instead of $e_{i} e_{i}=1$ as before, we now have $e_{i} e_{i}=e_{i} \cdot e_{i}$, as required.

If $\left\{f_{i}\right\}$ is another orthonormal basis, then by Sylvester's theorem, $f_{i} \cdot f_{i}=1$ for $p$ of the $f_{i}$ 's and $f_{i} \cdot f_{i}=-1$ for $q$ of the $f_{i}$ 's. Then Eq. (6) again shows that the $f$ 's have the same properties as the $e$ 's.

7. Other Constructions. Emil Artin has given an elegant and simple elementary construction of $\operatorname{GA}(n)[2$, p. 186]. Our construction is better motivated and, I think, somewhat simpler.

Marcel Riesz' construction in his Maryland lectures [6, Sec. 1.2-1.4] is incomplete. Riesz introduces products of the form Eq. (3) for orthonormal basis vectors $\left\{e_{i}\right\}$ and stipulates that $e_{i} e_{i}=1$ and $e_{i} e_{j}=-e_{j} e_{i}$ for $i \neq j$. (Our Eqs. (1) and (2).) But to show that these rules can be consistently applied, he needs something analogous to our lemma, which he does not supply. And once it is supplied, the associativity of the geometric product is trivial, as we have seen. Then Riesz's proof of the associativity in Sec. 1.3 becomes superfluous.

Ambjorn Naeve and Lars Svensson have given a construction which uses concepts from abstract algebra (rings, totally ordered sets, ideals) [5], although it could be reexpressed in more elementary terms.

R.D. Arthan has given a "minmalist" construction of the geometric algebra $[1] .^{3}$

Pertti Lounesto has catalogued several nonelementary constructions of $\mathrm{GA}(n)$, including those using a Grassman algebra, a tensor product, and a universal algebra [4]. These constructions obscure the simplicity and elementary nature of the geometric algebra.

Acknowledgment. I thank Martin Barrett for helpful comments.

\footnotetext{
3 Added 10/25/07.
} 


\section{References}

[1] R.D. Arthan, A Minimalist Construction of the Geometric Algebra, http: //arxiv.org/abs/math/0607190.

[2] E. Artin, Geometric Algebra, Interscience, New York, 1957.

[3] G. Isaacs, Real numbers; a development of the real numbers in an axiomatic set theory, McGraw-Hill, London, 1968.

[4] P. Lounesto, Marcel Riesz's Work on Clifford Algebras, in [6].

[5] A. Naeve and L. Svensson, Geo-MAP Unification, in Geometric Computing using Clifford Algebra, Ed. G. Sommer, Springer, 1999.

[6] M. Riesz, Clifford Numbers and Spinors, Kluwer Academic Publishers, Dordrecht, 1993. 